Homology, Homotopy and Applications, vol.7(2), 2005, pp.123-137

\title{
FREE HOMOTOPY ALGEBRAS
}

\author{
MARTIN MARKL
}

(communicated by Tom Lada)

\begin{abstract}
An explicit description of free strongly homotopy associative and free strongly homotopy Lie algebras is given. A variant of the Poincaré-Birkhoff-Witt theorem for the universal enveloping $\mathrm{A}(m)$-algebra of a strongly homotopy Lie algebra is formulated.
\end{abstract}

\section{Introduction}

This note was originated many years ago as my reaction to questions of several people how free strongly homotopy algebras can be described and what can be said about the structure of the universal enveloping $\mathrm{A}(m)$-algebra of an $\mathrm{L}(m)$ algebra constructed in $[\mathbf{3}]$, and then circulated as a "personal communication." I must honestly admit that it contains no really deep result and that everything I did was that I expanded definitions and formulated a couple of statements with more or less obvious proofs.

$\mathrm{A}(m)$-algebras and their strict homomorphisms [5, pages 147-148] form an equationally given algebraic category $\mathrm{A}(m)$. It follows from general theory that the forgetful functor to the category gVect of graded vector spaces, $\square: \mathrm{A}(m) \rightarrow$ gVect, has a left adjoint $\mathfrak{A}:$ gVect $\rightarrow \mathrm{A}(m)$. Given a graded vector space $X \in$ gVect, the object $\mathfrak{A}(X) \in \mathrm{A}(m)$ is the free $\mathrm{A}(m)$-algebra on the graded vector space $X$. We will also, for $n<m$, consider forgetful functors $\square: \mathrm{A}(m) \rightarrow \mathrm{A}(n)$ and their left adjoints $\mathfrak{m}_{\mathfrak{A}}^{n}: \mathrm{A}(n) \rightarrow \mathrm{A}(m)$; here the case $n=1$ is particularly important, because $\mathfrak{M}^{\mathfrak{A}^{1}}: \operatorname{dgVect} \rightarrow \mathrm{A}(m)$ describes the free $\mathrm{A}(m)$-algebra generated by a differential graded vector space.

We believe there is no need to emphasize the important rôle of free objects in mathematics. Each $\mathrm{A}(m)$-algebra is a quotient of a free one and free $\mathrm{A}(m)$ algebras were used in our definition of the universal enveloping algebra of a strongly homotopy Lie algebra [3, page 2154]. It could be useful to have a concrete description of these free algebras, as explicit as, for example, the description of free associative algebras by tensor algebras.

This brief note gives such a description in terms of planar trees. Replacing planar trees by non-planar ones, one can equally easy represent also free strongly homotopy Lie algebras. Surprisingly, these free algebras are simpler objects that their strict

This work was supported by the grant GA ČR 201/96/0310 and MŠMT ME603. Received June 17, 2004, revised November 29, 2004; published on April 22, 2005. 2000 Mathematics Subject Classification: 08B20.

Key words and phrases: Free algebra, A $(m)$-algebra, L $(m)$-algebra, PBW theorem. (C) 2005, Martin Markl. Permission to copy for private use granted. 
counterparts and admit a nice linear basis (Section 3), while free (strict) Lie algebras are very complicated objects (see, for example, $[\mathbf{9}$, Chapter IV $]$ ). This might be explained by the fact that axioms of strongly homotopy algebras are certain resolutions of strict axioms, thus they are more amenable. They manifest some properties of distributive laws [6] - they are 'directed,' therefore one can obtain unique representatives of elements of the corresponding operads in terms of admissible trees, like in Proposition 1.2, with obvious implications for the structure of free algebras.

In the first section of this note we describe the operad $\stackrel{m}{\mathcal{A}}$ for $\mathrm{A}(m)$-algebras ${ }^{1}$ and then, in Section 2, free $\mathrm{A}(m)$-algebras. In the third section we modify these constructions to free strongly homotopy Lie algebras. In the last section we prove a Poincaré-Birkhoff-Witt-type theorem for strongly homotopy Lie algebras and formulate a problem related to the structure of the category of modules. In the appendix we then recall the language of trees used throughout the paper.

\section{Operad for $\mathbf{A}(m)$-algebras}

In this note we assume a certain familiarity with operads, a good reference book for this subject is $[\mathbf{8}]$. For simplicity we suppose the ground ring $\mathbf{k}$ to be a field of characteristic zero, though the results of this and the following section remain true over an arbitrary ring.

Let $m$ be a natural number or $\infty$. Recall (see [10] for the original definition, but we use the sign convention of [5]) that an $\mathrm{A}(m)$-algebra is a graded $\mathbf{k}$-module $A$ together with a set $\left\{\mu_{k} ; 1 \leqslant k \leqslant m, k<\infty\right\}$ of degree $k-2$-linear maps, $\mu_{k}: \bigotimes^{k} A \rightarrow A$, such that

$$
\sum_{\lambda=0}^{n-1} \sum_{k=1}^{n-\lambda}(-1)^{\omega} \cdot \mu_{n-k+1}\left(a_{1}, \ldots, a_{\lambda}, \mu_{k}\left(a_{\lambda+1}, \ldots, a_{\lambda+k}\right), a_{\lambda+k+1}, \ldots, a_{n}\right)=0
$$

for all homogeneous $a_{1}, \ldots, a_{n} \in A, n \leqslant m$, where the sign is given as

$$
\omega:=k+\lambda+k \lambda+k\left(\left|a_{1}\right|+\ldots+\left|a_{\lambda}\right|\right) .
$$

It easily follows from the above axiom that $\mu_{1}: A \rightarrow A$ is a degree -1 differential. This operation will play a special rôle and we denote it by $\partial:=\mu_{1} . \mathrm{A}(m)$-algebras are algebras over a certain non- $\Sigma$ operad $\underline{\mathcal{A}}=\{\underline{\mathcal{A}}(n)\}_{n \geqslant 1}$ in the monoidal category of graded vector spaces. This operad can be constructed as follows.

Let $\stackrel{\stackrel{m}{\mathcal{F}}}{=}=\{\underline{\underline{F}}(n)\}_{n \geqslant 1}$ be the free graded non- $\Sigma$ operad $\underline{\Gamma}\left(\xi_{1}, \xi_{2}, \ldots, \xi_{m}\right)$ generated by operations $\xi_{i}, l \leqslant i \leqslant m$, where each $\xi_{i}$ has arity $i$ and degree $i-2$. Alternatively, let $\mathrm{m}(n)$ be the set of all connected directed planar trees with $n$ input edges, all of whose vertices have at most $m$ input edges; we admit also 'unary' vertices with only one input edge.

Let $\operatorname{Vert}(T)$ denote the set of vertices of a tree $T \in \stackrel{m}{T}(n)$. For $v \in \operatorname{Vert}(T)$, let $\operatorname{val}(v)$ be the number of input edges of $v$. We assign to each $T \in \stackrel{m}{\mathrm{~T}}(n)$ a degree

${ }^{1}$ underlining indicates that it is a non- $\Sigma$ operad 


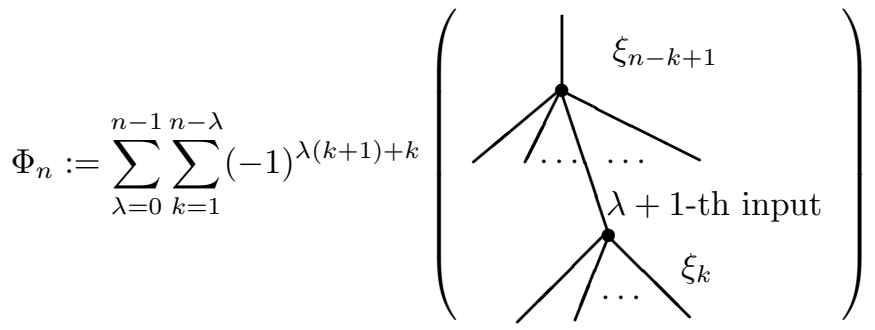

Figure 1: Relations of $\mathrm{A}(m)$-algebras in the tree language.

$\operatorname{deg}(T)$ by the formula

$$
\operatorname{deg}(T):=\sum_{v \in \operatorname{Vert}(T)}(\operatorname{val}(v)-2) .
$$

Then $\stackrel{\stackrel{m}{\mathcal{F}}}{(n)}$ can be naturally identified with the $\mathbf{k}$-linear span of the set $\stackrel{m}{\mathrm{~T}}(n)$,

$$
\underline{\stackrel{m}{\mathcal{F}}}(n)=\operatorname{Span}_{\mathbf{k}}(\stackrel{m}{\mathrm{~T}}(n)),
$$

see [8, Section II.1.9]. The operadic structure is, in this language, defined by grafting the corresponding trees. This means that, for $T \in \stackrel{m}{\mathrm{~T}}(a)$ and $S \in \stackrel{m}{\mathrm{~T}}(b)$, the operadic $i$-th circle product [8, Definition II.1.16] $T \circ_{i} S$ is the tree obtained by grafting the tree $S$ at the $i$-th input edge of $T$. Similarly, for $T \in \stackrel{m}{\mathrm{~T}}(l)$ and $S_{j} \in \stackrel{m}{\mathrm{~T}}\left(k_{j}\right), 1 \leqslant j \leqslant l$, the operadic composition [8, Definition II.1.4] $\gamma\left(T ; S_{1}, \ldots, S_{l}\right)=T\left(S_{1}, \ldots, S_{l}\right) \in$ $\mathrm{T}\left(k_{1}+\cdots+k_{l}\right)$ is the tree obtained by grafting $S_{j}$ at the $j$-th input edge of $T$, for each $1 \leqslant j \leqslant l$.

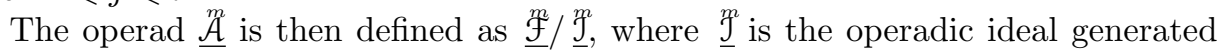
by the elements

$$
\Phi_{n}:=\sum_{\lambda=0}^{n-1} \sum_{k=1}^{n-\lambda}(-1)^{\lambda(k+1)+k} \xi_{n-k+1} \circ_{\lambda+1} \xi_{k} \in \underline{\stackrel{m}{\mathcal{F}}}(n), n \leqslant m .
$$

In the tree language of (1), the generators $\xi_{j}$ are represented by $j$-corollas (denoted by the same symbol) $\xi_{j} \in \stackrel{m}{\mathrm{~T}}(j)$; recall that the $j$-corolla is the unique tree having exactly one vertex. Relations (2) then represent certain linear combinations of trees, indicated in Figure 1. Elements of $\stackrel{m}{\mathcal{A}}(n)$ are equivalence classes of linear combinations of trees from $\mathrm{m}_{\mathrm{T}}^{\mathrm{m}}(n)$, modulo the relations of Figure 1. Surprisingly, there exists, for any $n \geqslant 1$, a very natural basis for the $\mathbf{k}$-vector space $\underline{\mathcal{A}}(n)$.

Definition 1.1. We say that a tree $T \in \stackrel{m}{\mathrm{~T}}(n)$ is admissible if all its unary vertices (i.e. vertices with just one input edge) are input vertices of the tree $T$.

Let us denote by $\operatorname{Adm}(n) \subset \stackrel{m}{\mathrm{~T}}(n)$ the subset consisting of admissible trees. Then the following proposition holds.

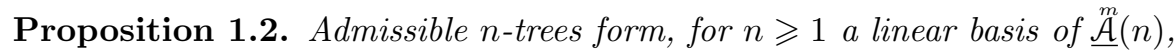

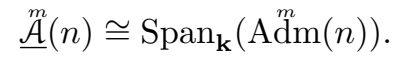


The proposition follows from the following lemma.

Lemma 1.3. There exists a unique map $\Omega: \stackrel{m}{\mathrm{~T}}(n) \rightarrow \operatorname{Span}_{\mathbf{k}}(\operatorname{Adm}(n))$ such that, for each $T \in \stackrel{m}{\mathrm{~T}}(n)$,

$$
\Omega(T) \equiv T \text { modulo the relations of Figure } 1 .
$$

Proof. If $T$ is admissible, we put $\Omega(T):=T$. If $T$ is not admissible, it contains a subtree of the form $\xi_{1} \circ_{1} \xi_{j}$, with some $1 \leqslant j \leqslant m$, or, pictorially,

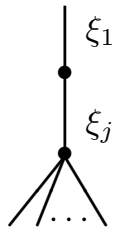

We may also assume that $j \geqslant 2$, otherwise $T \equiv 0$ modulo $\stackrel{m}{\mathcal{J}}$. Using the relations of Figure 1, we can replace the above subtree by a linear combination of trees of the form

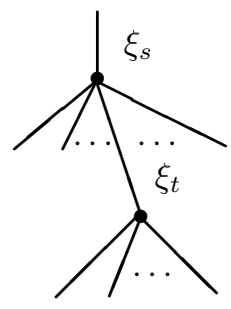

with $s \geqslant 2$. This enables us to move, by local replacements, the vertex $\xi_{1}$ towards the inputs of the tree. Repeating this process sufficiently many times, we get the requisite presentation $\Omega(T)$. It is easy to see that this presentation is unique, compare also similar arguments in the proof of [6, Theorem 2.3].

Proposition 1.2 immediately implies that

$$
\operatorname{dim}_{\mathbf{k}}(\underline{m}(n))=2^{n} \cdot b_{n}^{m}, \quad \text { for } m \leqslant 2, n \leqslant 1 .
$$

where $b_{n}^{m}$ is the number of all planar rooted reduced (i.e. without unary vertices) $n$-trees, all of whose vertices have arities $\leqslant m$. Clearly, $b_{n}^{m}$ equals the number of cells of the $m$-skeleton of Stasheff's associahedron $K_{n}$, compare also Example 2.2. Another kind of information about the size of the non- $\Sigma$-operad $\underline{\mathcal{A}}$ is given in:

Proposition 1.4. Let us consider the generating function

$$
\stackrel{m}{\varphi}(t):=\sum_{n \geqslant 1} \operatorname{dim}_{\mathbf{k}}(\underline{\underline{\mathcal{A}}}(n)) t^{n} .
$$

Then $\stackrel{1}{\varphi}^{(}(t)=2 t$ while, for $m \geqslant 2, \varphi(t)=\stackrel{m}{\varphi}(t)$ solves the equation

$$
\varphi(t)-2 t=\varphi^{2}(t)\left(1+\varphi(t)+\cdots+\varphi^{m-2}(t)\right) .
$$


Proof. Formula (4) is probably known, though we were not able to find a reference in the literature. We give a proof that can be easily modified to obtain the analogous formula (10) for $\mathrm{L}(m)$-algebras.

Let $d_{n}^{m}:=\operatorname{card}(\operatorname{Adm}(n))$. By Proposition $1.2, d_{n}^{m}:=\operatorname{dim}_{\mathbf{k}}(\underline{m}(n))$. Let us denote, just in this proof, by $\mathrm{T}_{n}$ the set of all planar reduced $n$-trees all of whose vertices have at most $m$ input edges. Let $r_{n}:=\operatorname{card}\left(\mathrm{T}_{n}\right)$. We claim that, for any $n \geqslant 2$,

$$
r_{n}=\sum_{i_{1}+i_{2}=n} r_{i_{1}} r_{i_{2}}+\sum_{i_{1}+i_{2}+i_{3}=n} r_{i_{1}} r_{i_{2}} r_{i_{3}}+\cdots+\sum_{i_{1}+\cdots+i_{m}=n} r_{i_{1}} \cdots r_{i_{m}}
$$

and

$$
d_{n}^{m}=2^{n} r_{n}
$$

The first equation follows from the decomposition

$$
\mathrm{T}_{n}=\mathrm{T}_{n, 2} \sqcup \mathrm{T}_{n, 3} \sqcup \cdots \sqcup \mathrm{T}_{n, m},
$$

where $\mathrm{T}_{n, i} \subset \mathrm{T}_{n}$ is the subset of trees whose root has $i$ input edges. The second equation follows from the observation that each admissible tree $T \in \operatorname{Adm}(n)$ can be obtained from a tree $S \in \mathrm{T}_{n}$ by grafting terminal unary trees at the input legs of $S$; there are $2^{n}$ ways to do this. If we denote $\psi(t):=\sum_{n \geqslant 1} r_{n} t^{n}$, then (5) implies

$$
\psi(t)-t=\psi^{2}(t)\left(1+\cdots+\psi^{m-2}(t)\right),
$$

while (6) implies that $\varphi(t)=\psi(2 t)$. This finishes the proof.

Example 1.5. For $m=\infty$, equation (4) can be rewritten as

$$
2 \varphi^{2}(t)-\varphi(t)(1+2 t)+2 t=0 .
$$

For $m=2$, (4) gives $\varphi(t)-2 t=\varphi^{2}(t)$, therefore

$$
\varphi(t)=\frac{1-\sqrt{(1-8 t)}}{2} .
$$

A little exercise in Taylor expansions results in

$$
d_{n}^{2}=\frac{2^{2 n-1}(2 n-3) ! !}{n !} \quad \text { for } n \geqslant 2,
$$

where as usual

$$
(2 n-3) ! !=1 \cdot 3 \cdot 5 \cdots(2 n-3) .
$$

\section{Free $\mathbf{A}(m)$-algebras}

The following theorem relies on the notation introduced in the previous section. Recall namely that $\operatorname{Adm}(n)$ denotes the set of admissible $n$-trees in the sense of

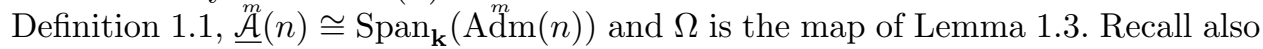
that we denoted by $\xi_{j}$ the $j$-corolla. If $T_{1}, \ldots, T_{j}$ are trees, then $\xi_{j}\left(T_{1}, \ldots, T_{j}\right)$ is the tree obtained by grafting the tree $T_{i}$ at the $i$-th input edge of the tree $\xi_{j}, 1 \leqslant i \leqslant j$. 
Theorem 2.1. Let $X$ be a graded vector space. Then the free $A(m)$-algebra $\stackrel{m}{\mathfrak{A}}(X)$ on $X$ can be described as $\stackrel{m}{\mathfrak{A}}(X)=\bigoplus_{n \geqslant 1} \stackrel{m}{\mathfrak{A}}^{n}(X)$, with

$$
\stackrel{m}{\mathfrak{A}}^{n}(X):=\underline{\stackrel{m}{\mathcal{A}}(n) \otimes X^{\otimes n} .}
$$

The structure operations $\mu_{1}, \ldots, \mu_{m}$ are defined as follows. Let $\vec{v}_{j} \in X^{\otimes k_{j}}$ and $T_{j} \in \operatorname{Adm}\left(k_{j}\right), 1 \leqslant j \leqslant k$. Then

$$
\mu_{k}\left(T_{1} \otimes \vec{v}_{1}, \ldots, T_{k} \otimes \vec{v}_{k}\right):=\Omega\left(\xi_{k}\left(T_{1}, \ldots, T_{k}\right)\right) \otimes\left(\vec{v}_{1} \otimes \cdots \otimes \vec{v}_{k}\right) .
$$

Proof. It follows from general theory [8, Section II.1.4] that the free $\underline{\mathcal{P}}$-algebra $F_{\mathcal{P}}(X)$ on a graded vector space $X$, where $\underline{\mathcal{P}}$ is a non- $\Sigma$ operad, is given by $F_{\mathcal{P}}(X)=$ $\bar{\bigoplus}_{n \geqslant 1} F_{\underline{\mathcal{P}}}^{n}(X)$, with $F_{\underline{\mathcal{P}}}^{n}(X):=\underline{\mathcal{P}}(n) \otimes X^{\otimes}$, with the algebra structure induced from the operad structure of $\underline{\mathcal{P}}$. Our theorem is then obtained by taking $\underline{\mathcal{P}}:=\underline{\underline{\mathcal{A}}}$ and applying the results on the structure of this operad proved in the previous section.

Observe that the free algebra $\mathfrak{A}(X)$ is bigraded. The internal grading is the grading of the underlying vector space, while the external grading is given by the decomposition

$$
\mathfrak{\mathfrak { A }}(X)=\bigoplus_{n \geqslant 1}^{\mathfrak{Q}^{n}} \mathfrak{A}^{n}(X)
$$

Because all main structures considered in this note (vector spaces, $\mathrm{A}(m)$-algebras, $\mathrm{L}(m)$-algebras, etc.) have an implicit internal grading, we will usually mean by saying that an object is graded the presence of an external grading. We believe that the actual meaning will always be clear from the context.

Example 2.2. There exists a simple way to encode elements of $\operatorname{Adm}(n)$. Let $\stackrel{m}{B}(n)$ be the set of all meaningful bracketings of strings $\epsilon_{1} \epsilon_{2} \cdots \epsilon_{n}$, where $\epsilon_{i} \in\{\circ, \bullet\}$ and no pair of brackets encompasses more than $m$ terms. A moment's reflection shows that $\operatorname{Adm}(n) \cong \stackrel{m}{B}(n), n \geqslant 1$. Thus

$$
\begin{aligned}
& \operatorname{Adm}(1)=\{\circ, \bullet\}, m \geqslant 1, \\
& \operatorname{Adm}(2)=\emptyset, \operatorname{Adm}(2)=\{(\circ \circ),(\circ \bullet),(\bullet \circ),(\bullet \bullet)\}, m \geqslant 2, \\
& \operatorname{Adm}(3)=\emptyset, \\
& \operatorname{Adm}^{2}(3)=\{((\circ \circ) \circ),((\bullet \circ) \circ),((\bullet \bullet) \circ), \ldots,(\circ(\circ \circ)),(\bullet(\circ \circ)),(\bullet(\bullet \circ)), \ldots\}, \\
& \operatorname{Adm}_{m}^{m}(3)=\operatorname{Adm}(3) \cup\{(\circ \circ \circ),(\bullet \circ \circ),(\bullet \bullet \circ), \ldots\}, m \geqslant 3, \text { etc. }
\end{aligned}
$$

We conclude that $\mathfrak{A}(X)$ is spanned by all meaningful bracketings of strings of "variables" $x$ and $\partial x, \operatorname{deg}(\partial x)=\operatorname{deg}(x)-1, x \in X$, with no pair of brackets encompassing more than $m$ terms. Thus $\mathfrak{A}(X)=\mathfrak{A}^{1}(X)$ is the free differential complex generated by $X, \mathfrak{A}(X) \cong X \oplus \partial X$. Similarly,

$$
\begin{aligned}
\mathfrak{A}^{2}(X) \cong(X \otimes X) \oplus(\partial X \otimes X) \oplus(X \otimes \partial X) \oplus(\partial X \otimes \partial X), \text { for } m \geqslant 2, \\
\mathfrak{A}^{2}(X) \cong((X \otimes X) \otimes X) \oplus((\partial X \otimes X) \otimes X) \oplus((X \otimes \partial X) \otimes X) \\
\oplus((X \otimes X) \otimes \partial X) \oplus((\partial X \otimes \partial X) \otimes X) \oplus((\partial X \otimes X) \otimes \partial X)
\end{aligned}
$$




$$
\begin{aligned}
&((X \otimes \partial X) \otimes \partial X) \oplus((\partial X \otimes \partial X) \otimes \partial X) \oplus(X \otimes(X \otimes X)) \\
&(\partial X \otimes(X \otimes X)) \oplus(X \otimes(\partial X \otimes X)) \oplus(X \otimes(X \otimes \partial X)) \\
& \oplus(\partial X \otimes(\partial X \otimes X)) \oplus(\partial X \otimes(X \otimes \partial X)) \oplus(X \otimes(\partial X \otimes \partial X)) \\
& \oplus(\partial X \otimes(\partial X \otimes \partial X)) \\
& \mathfrak{A}^{3}(X) \cong \mathfrak{A}^{2}(X) \oplus(X \otimes X \otimes X) \oplus(\partial X \otimes X \otimes X) \oplus(X \otimes \partial X \otimes X) \oplus(X \otimes X \otimes \partial X) \\
& \oplus(\partial X \otimes \partial X \otimes X) \oplus(\partial X \otimes X \otimes \partial X) \oplus(X \otimes \partial X \otimes \partial X) \\
& \oplus(\partial X \otimes \partial X \otimes \partial X), \text { for } m \geqslant 3, \text { etc. }
\end{aligned}
$$

We see that

$$
\operatorname{dim}\left(\mathfrak{\mathfrak { A }}^{n}(X)\right)=(2 \operatorname{dim}(X))^{n} \cdot b_{n}^{m}, n \geqslant 1, m \geqslant 2,
$$

where $b_{n}^{m}$ is as in (3).

Unital $\mathrm{A}(m)$-algebras. Recall [5, page 148] that an $\mathrm{A}(m)$-algebra $A=\left(A,\left\{\mu_{n}\right\}\right)$ is unital if there exists an element $1=1_{A} \in A$ such that $\mu_{2}\left(a, 1_{A}\right)=\mu_{2}\left(1_{A}, a\right)=a$, for all $a \in A$, and that $\mu_{n}\left(a_{1}, \ldots, a_{i-1}, 1_{A}, a_{i+1}, \ldots, a_{n}\right)=0$, for all $n \neq 2$ and $1 \leqslant i \leqslant n$. The free unital $A(m)$-algebra can be constructed as $\operatorname{Span}_{\mathbf{k}}(1) \oplus \mathfrak{A}(X)$, with the structure operations defined in an obvious way.

Important modifications. One may as well consider, for any $n<m$, the forgetful functor $\square: \mathrm{A}(m) \rightarrow \mathrm{A}(n)$. This functor has a left adjoint $\stackrel{m i n}{\mathfrak{A}}: \mathrm{A}(n) \rightarrow \mathrm{A}(m)$ which can be described as follows. Given an $\mathrm{A}(n)$-algebra $\left(V, m_{1}, m_{2}, \ldots, m_{n}\right)$, consider the free $\mathrm{A}(m)$-algebra $\mathfrak{A}(V)$ on the graded vector space $V$, and let $\mu_{1}, \ldots, \mu_{m}$ be the structure operations of $\mathfrak{A}(V)$. Then

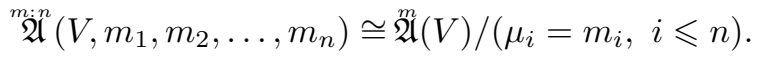

A very important special case is $n=1$ when we obtain a left adjoint $\stackrel{m}{\mathfrak{A}} \cdot 1: \operatorname{dgVect} \rightarrow$ $\mathrm{A}(m)$ to the forgetful functor $\square: \mathrm{A}(m) \rightarrow$ dgVect to the category of differential graded vector spaces. One can easily verify that

$$
\stackrel{m \cdot 1}{\mathfrak{A}}(V, \partial) \cong \bigoplus_{n \geqslant 1}(\underline{\mathfrak{A}}(n), \partial) \otimes(V, \partial)^{\otimes n},
$$

where $(\underline{\mathscr{A}}, \partial)$ is now the non- $\Sigma$ operad describing $\mathrm{A}(m)$-algebras as algebras in the monoidal category of differential vector spaces. Let us recall [7, page 1493] that $\underline{\stackrel{m}{\mathcal{A}}}=\underline{\Gamma}\left(\xi_{2}, \ldots, \xi_{m}\right)$ with the differential

$$
d\left(\xi_{n}\right):=\sum(-1)^{(b+1)(i+1)+b} \cdot \xi_{a} \circ_{i} \xi_{b},
$$

where the summation runs over all $a, b \geqslant 2$ with $a+b=n+1$ and $1 \leqslant i \leqslant a$. Formula (7) is in a perfect harmony with [8, Definition II.1.24] describing free operad algebras in a general monoidal category.

Example 2.3. Clearly $\stackrel{m}{\mathfrak{A}}^{1}(V, \partial) \cong(V, \partial)$, and, for $m \geqslant 2,{\stackrel{m}{\mathfrak{A}} \mathfrak{A}^{2}}^{2}(V, \partial) \cong(V \otimes V)$ with the induced differential. As graded vector spaces

$$
\begin{aligned}
\mathfrak{\mathfrak { A }}^{2} \mathbf{1}^{3}(V, \partial) & \cong((V \otimes V) \otimes V) \oplus(V \otimes(V \otimes V)), \\
\mathfrak{A}^{m \cdot 1}(V, \partial) & \cong \mathfrak{A}^{2: 1}(V) \oplus(V \otimes V \otimes V), \text { for } m \geqslant 3,
\end{aligned}
$$




$$
\begin{aligned}
& \mathfrak{\mathfrak { A }}^{2} \mathbf{1}^{4}(V, \partial) \cong(((V \otimes V) \otimes V) \otimes V) \oplus(V \otimes(V \otimes(V \otimes V))) \oplus((V \otimes V) \otimes(V \otimes V)) \\
& \oplus((V \otimes(V \otimes V)) \otimes V) \oplus(V \otimes((V \otimes V) \otimes V)), \\
& \mathfrak{\mathfrak { A }}^{3} \mathbf{i}^{4}(V, \partial) \cong \mathfrak{\mathfrak { A }}^{2} \mathbf{1}^{4}(V, \partial) \oplus((V \otimes V \otimes V) \otimes V) \oplus(V \otimes(V \otimes V \otimes V)) \oplus((V \otimes V) \otimes V \otimes V) \\
& \oplus(V \otimes(V \otimes V) \otimes V) \oplus(V \otimes V \otimes(V \otimes V)), \\
& \stackrel{m}{\mathfrak{A}}^{1} 4(V, \partial) \cong \mathfrak{\mathfrak { A }}^{3 \mathbf{1}^{4}}(V, \partial) \oplus(V \otimes V \otimes V \otimes V \otimes V) \text { for } m \geqslant 4 \text {, etc. }
\end{aligned}
$$

The action of the differential in $\stackrel{m}{\mathfrak{A}}^{1}(V, \partial)$ can be easily read off from the axioms of $\mathrm{A}(m)$-algebras, for example

$$
\begin{aligned}
\partial(x \otimes y \otimes z)=((x \otimes y) \otimes z) & -(x \otimes(y \otimes z)) \\
& -(\partial x \otimes y \otimes z)-(-1)^{|a|}(x \otimes \partial y \otimes z)-(-1)^{|a|+|b|}(x \otimes y \otimes \partial z),
\end{aligned}
$$

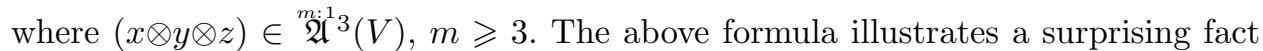
that the differential in $\stackrel{\mathfrak{A}}{\mathfrak{A}}(V, \partial)$ is nontrivial even if the differential $\partial$ on $V$ is zero, because in this case still

$$
\partial(x \otimes y \otimes z)=((x \otimes y) \otimes z)-(x \otimes(y \otimes z)) !
$$

The dimension of the homogeneous components of $\stackrel{m}{\mathfrak{A}}^{1}(V, \partial V)$ clearly equals

$$
\operatorname{dim} \stackrel{m \cdot 1}{\mathfrak{A}}{ }^{n}(V, \partial)=(\operatorname{dim}(V))^{n} \cdot b_{n}^{m}, \text { for } n \geqslant 1, m \geqslant 2,
$$

where the integers $b_{n}^{m}$ are as in (3).

\section{Free strongly homotopy Lie algebras}

In this section we indicate how to modify previous results to strongly homotopy Lie algebras. Recall ([4] or [3, Definition 2.1]) that an $\mathrm{L}(m)$-structure on a graded vector space $L$ is a system $\left\{l_{k} ; 1 \leqslant k \leqslant m, k<\infty\right\}$ of degree $k-2$ linear maps $l_{k}: \otimes{ }^{k} L \rightarrow L$ which are antisymmetric in the sense that

$$
l_{k}\left(x_{\sigma(1)}, \ldots, x_{\sigma(k)}\right)=\chi(\sigma) l_{k}\left(x_{1}, \ldots, x_{n}\right)
$$

for all $\sigma \in \Sigma_{n}$ and $x_{1}, \ldots, x_{n} \in L$. Here $\chi(\sigma):=\operatorname{sgn}(\sigma) \cdot \epsilon(\sigma)$, where $\epsilon(\sigma)$ is the Koszul sign of the permutation $\sigma$, see [3, page 2148] for details. Moreover, the following generalized form of the Jacobi identity is supposed to be satisfied for any $n \leqslant m:$

$$
\sum_{i+j=n+1} \sum_{\sigma} \chi(\sigma)(-1)^{i(j-1)} l_{j}\left(l_{i}\left(x_{\sigma(1)}, \ldots, x_{\sigma(i)}\right), x_{\sigma(i+1)}, \ldots, x_{\sigma(n)}\right)=0,
$$

where the summation is taken over all $(i, n-i)$-unshuffles with $i \geqslant 1$.

The operad $\stackrel{m}{\mathcal{L}}=\{\stackrel{m}{\mathcal{L}}(n)\}_{n \geqslant 1}$ describing $\mathrm{L}(m)$-algebras as algebras in the category of graded vector spaces can be constructed as follows. Let $\stackrel{m}{\mathcal{F}}=\{\stackrel{m}{\mathcal{F}}(n)\}_{n} \geqslant 1$ be the free graded $\Sigma$-operad $\Gamma\left(\zeta_{1}, \zeta_{2}, \ldots, \zeta_{m}\right)$ generated by the operations $\zeta_{i}, l \leqslant i \leqslant m$, where each $\zeta_{i}$ has $i$ inputs, degree $i-2$ and spans the signum representation of the symmetric group $\Sigma_{i}$. Using trees, this operad can be described as follows. Let $\mathrm{T}(n)$ be now the set of all directed labeled (non-planar, abstract) trees with $n$-input edges, all of whose vertices have at most $m$ input edges. We again admit also 'unary' 


$$
0=\left.\sum_{i+j=n+1} \sum_{\sigma} \chi(\sigma)(-1)^{i(j-1)} \cdot\right|_{\sigma(1)} ^{(2)}
$$

Figure 2: Relations for strongly homotopy Lie algebras in the tree language.

vertices with only one input edge and 'labeled' means that the input legs are labeled by natural numbers $1, \ldots, n$. Then, as before,

$$
\mathcal{F}(n) \cong \operatorname{Span}_{\mathbf{k}}(\stackrel{m}{\mathrm{~T}}(n)) .
$$

We put $\stackrel{m}{\mathcal{L}}:=\stackrel{m}{\mathcal{F}} / \mathcal{m}$, where the operadic ideal $\stackrel{m}{\mathcal{J}}$ is now generated by relations whose pictorial presentation is indicated in Figure 2.

By similar arguments as in the previous sections we easily infer that there is a natural basis of the $\mathbf{k}$-vector space $\stackrel{m}{\mathcal{L}}(n)$, formed by the set $\operatorname{Adm}(n)$ of admissible $n$-trees, whose definition is formally the same as in Definition 1.1, only removing everywhere the adjective "planar." The size of the operad $\stackrel{m}{\mathcal{L}}$ is described in the following proposition whose proof is similar to that of Proposition 1.4.

Proposition 3.1. The generating function $\vartheta(t)=\stackrel{m}{\vartheta}(t):=\sum_{n \geqslant 1} \frac{1}{n !} \operatorname{dim}_{\mathbf{k}}(\stackrel{m}{\mathcal{L}}(n)) t^{n}$ satisfies the equation

$$
\vartheta(t)-2 t=\vartheta^{2}(t)\left(\frac{1}{2 !}+\frac{1}{3 !} \vartheta(t)+\cdots+\frac{1}{m !} \vartheta^{m-2}(t)\right) .
$$

Example 3.2. For $m=\infty$, equation (10) becomes $2 \vartheta(t)-2 t-1=\exp (\vartheta(t))$. For $m=2$, equation (10) says that $\vartheta(t)=1-\sqrt{1-4 t^{2}}$, therefore

$$
\operatorname{dim}_{\mathbf{k}}(\stackrel{2}{\mathcal{L}}(n))=2^{n} \cdot(2 n-3) ! !, n \geqslant 1 .
$$

As in Lemma 1.3, we have a map $\Omega: \stackrel{m}{\mathrm{~T}}(n) \rightarrow \operatorname{Span}_{\mathbf{k}}(\operatorname{Adm}(n))$ with the property that $\Omega(T) \equiv T$ modulo relations of Figure 2 . Free $\mathrm{L}(m)$-algebras are then described as follows.

Theorem 3.3. Let $Y$ be a graded vector space. The free $\mathrm{L}(m)$-algebra $\stackrel{m}{\mathfrak{L}}(Y)$ generated by $Y$ can defined as $\stackrel{m}{\mathfrak{L}}(Y)=\bigoplus_{n \geqslant 1} \stackrel{m}{\mathfrak{L}} n^{n}(Y)$, where

$$
\stackrel{m}{\mathfrak{L}}^{n}(Y):=\stackrel{m}{\mathcal{L}}(n) \otimes_{\Sigma_{n}} Y^{\otimes n} .
$$

The structure operations $l_{1}, \ldots, l_{m}$ are given by the following rule. Let $\vec{v}_{j} \in Y^{\otimes k_{j}}$ and $T_{j} \in \operatorname{Adm}\left(k_{j}\right), 1 \leqslant j \leqslant k$. Then

$$
l_{k}\left(T_{1} \otimes_{\Sigma_{k_{1}}} \vec{v}_{1}, \ldots, T_{k} \otimes_{\Sigma_{k_{1}}} \vec{v}_{k}\right):=\Omega\left(\xi_{k}\left(T_{1}, \ldots, T_{k}\right)\right) \otimes_{\Sigma_{k_{1}+\cdots+k_{k}}}\left(\vec{v}_{1} \otimes \cdots \otimes \vec{v}_{k}\right) .
$$


Remark 3.4. In exactly the same fashion as for $\mathrm{A}(m)$-algebras one may define left adjoints $\stackrel{m: n}{\mathcal{L}}: \mathrm{L}(n) \rightarrow \mathrm{L}(m)$ to the forgetful functor $\square: \mathrm{L}(m) \rightarrow \mathrm{L}(n), n<m$, as

$$
\stackrel{m: n}{\mathcal{L}}\left(L, \lambda_{1}, \lambda_{2}, \ldots, \lambda_{n}\right)=\stackrel{m}{\mathfrak{L}}(L) /\left(\lambda_{i}=l_{i}, i \leqslant n\right),
$$

where $l_{i}$ are the structure operations of $\stackrel{m}{\mathfrak{L}}(L)$.

\section{Reflections on the PBW theorem}

As in the case of ordinary Lie algebras, there exists the symmetrization functor from the category $\mathrm{A}(m)$ of $\mathrm{A}(m)$-algebras to the category $\mathrm{L}(m)$ of $\mathrm{L}(m)$-algebras. More precisely, we proved in [3, Theorem 3.1]:

Theorem 4.1. Any $A(m)$-structure $\left\{\mu_{n}: \bigotimes^{n} V \longrightarrow V\right\}$ on a differential graded vector space $V$ induces an $L(m)$-structure $\left\{l_{n}: \otimes^{n} V \longrightarrow V\right\}$ on the same differential graded vector space, with

$$
l_{n}\left(v_{1} \otimes \ldots \otimes v_{n}\right):=\sum_{\sigma \in \mathcal{S}_{n}} \chi(\sigma) \mu_{n}\left(v_{\sigma(1)} \otimes \ldots \otimes v_{\sigma(n)}\right), 2 \leqslant n \leqslant m .
$$

This correspondence defines a functor (the symmetrization) $(-)_{L}: \mathrm{A}(m) \longrightarrow \mathrm{L}(m)$.

In [3, Theorem 3.3], we also proved that:

Theorem 4.2. There exists a functor $\mathcal{U}_{m}: \mathrm{L}(m) \longrightarrow \mathrm{A}(m)$ that is left adjoint to the functor $(-)_{L}: \mathrm{A}(m) \longrightarrow \mathrm{L}(m)$.

The algebra $\mathcal{U}_{m}(L)$ of the previous theorem is called the universal enveloping A $(m)$-algebra of the $\mathrm{L}(m)$-algebra $L$. We gave, in [3, page 2154], the following explicit construction of $\mathcal{U}_{m}(L)$.

Start with the free unital $\mathrm{A}(m)$-algebra $\mathfrak{A}(L)$ generated by the underlying vector space $L$ of the $\mathrm{L}(m)$-algebra $L=\left(L,\left\{l_{n}\right\}\right)$ and let $\left\{\mu_{n}\right\}$ be the $\mathrm{A}(m)$-structure maps of $\mathfrak{A}(L)$. Let $I$ denote the $\mathrm{A}(m)$-ideal in $\mathfrak{A}(L)$ generated by the relations

$$
\sum_{\sigma \in S_{n}} \chi(\sigma) \mu_{n}\left(\xi_{\sigma(1)}, \ldots, \xi_{\sigma(n)}\right)=l_{n}\left(\xi_{1}, \ldots, \xi_{n}\right), \text { for } \xi_{1}, \ldots, \xi_{n} \in L, n \leqslant m .
$$

Then we put $\mathcal{U}_{m}(L):=\mathfrak{m}(L) / I$. This universal enveloping $\mathrm{A}(m)$-algebra is equipped with the canonical map (in fact, an inclusion) $\iota: L \rightarrow \mathcal{U}_{m}(L)$.

Remark 4.3. Hinich and Schechtman introduced in [2], for any $\mathrm{L}(m)$-algebra $L$ (they did it for $m=\infty$, but the general case is an obvious modification), an associative algebra (which they also called the universal enveloping algebra), having the property that the category of modules (in a suitable sense, see for example [3, Definition 5.1]) over an $\mathrm{L}(m)$-algebra $L$ is equivalent to the category of left modules over the universal enveloping associative algebra of $L$.

It is unclear whether there exists a functor $\mathcal{G}: \mathrm{L}(m) \rightarrow \mathrm{A}(m)$ such that the category of modules over an $\mathrm{L}(m)$-algebra $L$ would be naturally equivalent to the category of left modules, in the sense of [5, page 157], over the A $(m)$-algebra $\mathcal{G}(L)$. Our universal enveloping $\mathrm{A}(m)$-algebra functor whose definition we recalled above 
probably does not have this property. While it can be easily shown that each left module over the $\mathrm{A}(m)$-algebra $\mathcal{U}_{m}(L)$ naturally defines a module over $L$, we doubt that each $L$-module uniquely extends into a left module over $\mathcal{U}_{m}(L)$. The classical universal enveloping associative algebra of a strict Lie algebra is constructed as a left adjoint to the symmetrization functor. By a miracle, this functor also happens to describe the category of $L$-modules, but this mystic phenomenon does not take place in the category of $\mathrm{L}(m)$-algebras.

Moreover, there is no reason to believe that such a functor $\mathcal{G}$ exits. The category of modules over a given $\mathrm{L}(m)$-algebra $L$ is a well-behaved abelian category, therefore it should be the category of modules over an associative algebra, which turns out to be the one constructed by Hinich and Schechtman. On the other hand, we do not see why this abelian category should be at the same time the category of left modules over some $\mathrm{A}(m)$-algebra. Let us close this remark with

Problem 4.4. Let $L$ be an $\mathrm{L}(m)$-algebra. Does there exist an $\mathrm{A}(m)$-algebra $A$ with the property that the category of modules over $L$ is equivalent to the category of left modules over A?

The simplest form of the $\mathrm{P}$ (oincaré)-B(irkhoff)-W(itt) theorem for an ordinary Lie algebra $L=(L,[-,-])$ says that the associated graded algebra $G^{*}(L)$ of the universal enveloping algebra $\mathcal{U}(L)$ of $L$ is isomorphic to the free commutative associative algebra $S^{*}(L)$ (the polynomial ring) on the vector space $L$. More precisely, recall that

$$
\mathcal{U}(L)=T(L) /(x \otimes y-y \otimes x=[x, y], x, y \in L),
$$

where $T(L)$ is the free associative algebra (tensor algebra) on the vector space $L$. The algebra $\mathcal{U}(L)$ is filtered, the ascending filtration being given by $\mathcal{U}_{p}(L):=$ vector space generated by linear combinations of elements which can be presented as a product of $\leqslant p$ elements in the augmentation ideal of $T(L)$. Clearly $\mathcal{U}_{0}(L)=\mathbf{k}$ and $\mathcal{U}_{1}(L)=\mathbf{k} \oplus L$. If $G^{*}(L)$ denotes the associated graded algebra, then one proves that the natural map $L=G^{1}(L) \rightarrow G^{*}(L)$ is a monomorphism and $G^{*}(L)$ is generated by $L$. It is immediate to see that $x y=y x$, for arbitrary $x, y \in L \subset G^{*}(L)$. We formulate the following trivial observation.

Observation 4.5. Suppose that an associative algebra $A$ admits a set of mutually commutative generators. Then $A$ is commutative.

From this observation we infer that $G^{*}(L)$ is commutative, therefore there exists, by the universal property, a commutative algebra map $S^{*}(L) \rightarrow G^{*}(L)$, induced by the inclusion $L \subset G^{*}(L)$. The $\mathrm{PBW}^{2}$ theorem says that this map is an isomorphism of graded commutative associative algebras.

Let us try to guess which form the PBW theorem for the universal enveloping A $(m)$-algebra $\mathcal{U}_{m}(L)$ of an $\mathrm{L}(m)$-algebra $L$ may possibly have. The classical PBW theorem compares the associated graded of $\mathcal{U}(L)$ to a commutative associative

${ }^{2}$ not to be mistaken with Praha-Berlin-Warszawa cycling race 
algebra. Commutative associative algebras occur in the symbolic exact sequence (which may be found in [1, page 228])

Commutative associative algebras $\subset$ Associative algebras $\stackrel{\pi}{\longrightarrow}$ Lie algebras.

Here the 'projection' $\pi$ is given by the symmetrization of the associative product. Commutative associative algebras are also the quadratic duals of Lie algebras (and vice versa) [1, Theorem 2.1.11], but this coincidence is misleading. Summing up, the classical PBW theorem says that the associated graded $G^{*}(L)$ is the free algebra in the kernel of the symmetrization map $\pi$.

One would naturally expect something similar also in the $\mathrm{L}(m)$-algebra case. The kernel of the symmetrization map $(-)_{L}$ of Theorem 4.1 clearly consists of $\mathrm{A}(m)$ algebras $A=\left(A,\left\{\mu_{n}\right\}\right)$ such that

$$
\sum_{\sigma \in S_{n}} \chi(\sigma) \mu_{n}\left(a_{\sigma(1)}, \ldots, a_{\sigma(n)}\right)=0, n \geqslant 2, a_{1}, \ldots, a_{n} \in A .
$$

Therefore one tends to believe that $\mathrm{A}(m)$-algebras enjoying this form of symmetry are analogs of the polynomial algebra $S^{*}(L)$ from the classical PBW theorem. This is, however, not exactly so.

To see why, let $L=\left(L,\left\{l_{n}\right\}\right)$ be an $\mathrm{L}(m)$-algebra and define inductively an ascending filtration of the universal enveloping $\mathrm{A}(m)$-algebra $\mathcal{U}_{m}(L)$ by $\mathcal{U}_{m, 0}(L):=$ $\mathbf{k}, \mathcal{U}_{m, 1}(L):=\mathcal{U}_{m, 0} \cup \iota(L)$ and $\mathcal{U}_{m, p}(L)$ being the vector space generated by elements of the form $\mu_{k}\left(x_{1}, \ldots, x_{k}\right), k \geqslant 2, x_{i} \in \mathcal{U}_{m, p_{i}}(L)$ for $1 \leqslant i \leqslant k$ and $\sum_{1}^{k} p_{i} \leqslant p$. The associated graded $\mathrm{A}(m)$-algebra $G_{m}^{*}(L)$ is defined by

$$
G_{m}^{*}(L)=\bigoplus_{q \geqslant 0} G_{m}^{q}(L), G_{m}^{q}(L):=\mathcal{U}_{m, q}(L) / \mathcal{U}_{m, q-1}(L)
$$

with the $\mathrm{A}(m)$-structure maps induced by that of $\mathcal{U}_{m}(L)$. It is easily seen that $G_{m}^{*}(L)$ is generated by the image of the canonical inclusion $\iota: L \hookrightarrow \mathcal{U}_{m, 1}(L) \rightarrow$ $G_{m}^{*}(L)$. A moment's reflection shows that the elements of $L \subset G_{m}^{*}(L)$ 'mutually commute' in the sense that (11) is satisfied for $a_{1}, \ldots, a_{n} \in L$. Surprisingly, this does not imply that (11) is satisfied for all elements of $G_{m}^{*}(L)$ - there is no analog of Observation 4.5 for $\mathrm{A}(m)$-algebras! One must instead consider $\mathrm{A}(m)$-algebras $S_{m}(V, \partial)$ defined as

$$
S_{m}(V, \partial):=\stackrel{m: 1}{\mathfrak{A}}(V, \partial) / \mathfrak{I}_{m},
$$

where $\mathfrak{I}_{m}$ is the ideal generated by the relations

$$
\sum_{\sigma \in S_{n}} \chi(\sigma) \mu_{n}\left(v_{\sigma(1)}, \ldots, v_{\sigma(n)}\right)=0 \text { for } 2 \leqslant n \leqslant m \text { and } v_{1}, \ldots, v_{n} \in V .
$$

The algebra $S_{m}(V, \partial)$ is (externally) graded, with the grading induced from the (external) grading of the free $\mathrm{A}(m)$-algebra $\stackrel{m}{\mathfrak{A}}^{1}(V, \partial)$. By a miracle, $S_{m}^{*}(V, \partial)$ has the following very explicit description.

Choose a basis $\left\{f_{i}\right\}_{i \in I}$ of $V$ indexed by a linearly ordered set $I$. Let ${ }^{m}(V)$ be the set of all planar rooted reduced $n$-trees $S$ whose vertices have arity $\leqslant m$. We moreover suppose that the input legs of $S$ are labeled by elements of the basis $\left\{f_{i}\right\}_{i \in I}$ and that the labels satisfy the following condition: 
Let $v$ be an input vertex of $S$ and let $e_{1}, \ldots, e_{k}$ be the input legs of $v$, written in the order induced by the imbedding of $S$ into the plane. Let $f_{i_{1}}, \ldots, f_{i_{k}}$ be the corresponding labels. Then it is not true that $i_{1} \geqslant i_{2} \geqslant \cdots \geqslant i_{k}$.

Proposition 4.6. For each $n \geqslant 1, S_{m}^{n}(V) \cong \operatorname{Span}_{\mathbf{k}}\left(\stackrel{m}{T}^{n}(V)\right)$.

Proof. It follows from (7) that $\stackrel{m}{\mathfrak{A}}(V, \partial)$ is spanned by the set of planar directed reduced $n$-trees with all vertices of arity $\leqslant m$ and the input legs labeled by the chosen basis $\left\{f_{i}\right\}_{i \in I}$ of $V$. Let $v$ be an input vertex of $S, e_{1}, \ldots, e_{k}$ input legs of $v$ written in the order induced by the plane and $f_{i_{1}}, \ldots, f_{i_{k}}$ their labels.

Suppose $i_{1} \geqslant i_{2} \geqslant \cdots \geqslant i_{k}$. If $i_{1}=i_{k}$, then clearly $S \equiv 0$ modulo $\mathfrak{I}_{m}$. If $i_{1} \neq i_{k}$, we may replace $S$ modulo $\mathfrak{I}_{m}$ by a sum of trees that differ from $S$ only by labels of $e_{1}, \ldots, e_{k}$ which are changed to $i_{\sigma(1)}, \ldots, i_{\sigma(k)}$, for some $\sigma \in \Sigma_{k}$ such that it is not true that $i_{\sigma(1)} \geqslant i_{\sigma(2)} \geqslant \cdots \geqslant i_{\sigma(k)}$. Repeating this process at any input vertex of $S$, we replace $S$ modulo $\mathfrak{I}_{m}$ by a sum of trees from $\mathrm{T}^{n}(V)$. This replacement is obviously unique.

Let us formulate our version of the PBW theorem for the universal enveloping $\mathrm{A}(m)$-algebra of an $\mathrm{L}(m)$-algebra.

Theorem 4.7. The canonical map $\iota: L \hookrightarrow \mathcal{U}_{m, 1}(L) \rightarrow G_{m}^{*}(L)$ induces an isomorphism

$$
\rho: S_{m}^{*}(L) \cong G_{m}^{*}(L)
$$

of (externally) graded $A(m)$-algebras.

Proof. The proof relies on the fact that $\left\{\rho(S) ; S \in \mathrm{T}^{n}(V)\right\}$ forms a basis of $G_{m}^{n}(V)$, which easily follows from the above analysis. We leave the details to the reader.

Example 4.8. Let us analyze the case $m=2$ when $\mathrm{L}(m)$-algebras are just differential graded anti-commutative non-associative algebras. For an L(2)-algebra $L=(L,[-,-], \partial), \mathfrak{A}^{2} \mathfrak{l}(L)$ is the free non-associative dg algebra generated by $(L, \partial)$, the universal enveloping $\mathrm{A}(2)$-algebra of $L$ is given by

$$
\mathcal{U}_{2}(L)=\stackrel{2: 1}{\mathfrak{A}} /\left(x y-(-1)^{|x||y|} y x=[x, y], x, y \in L\right),
$$

and the associated graded $G_{2}^{*}(L)$ is the quotient of $\mathfrak{\mathfrak { A }}^{2}(L)$ by the ideal generated by $x y=(-1)^{|x||y|} y x$, for $x, y \in L$.

Remark 4.9. An ordinary strict Lie algebra $L=(L,[-,-])$ can be considered, for any $m \geqslant 2$, as an $\mathrm{L}(m)$-algebra with $l_{2}=[-,-]$ and $l_{n}=0$ if $n \neq 2$. Its universal enveloping $\mathrm{A}(m)$-algebra $\mathcal{U}_{m}(L)$ is a fully-fledged $\mathrm{A}(m)$-algebra, therefore an object completely different from the ordinary universal enveloping associative algebra $\mathcal{U}(L)$. Thus our $\mathcal{U}_{m}(L)$ cannot be considered as a generalization of the classical universal enveloping algebra functor. 


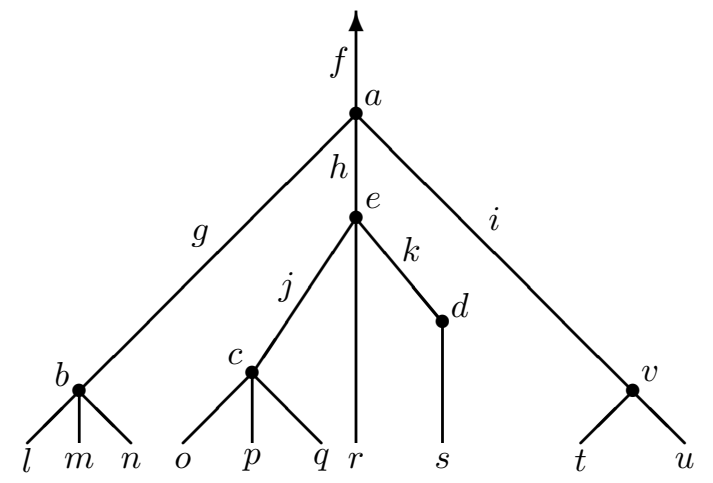

Figure 3: Language of trees: a 10-tree.

\section{Appendix: the tree language}

The following definitions can be found for example in [8, Section II.1.5]. By a tree we mean a connected graph $T$ without loops. Let $\operatorname{Vert}(T)$ denote the set of vertices of $T$. A valence of a vertex $v \in \operatorname{Vert}(T)$ is the number of edges adjacent to $v$. A leg of $T$ is an edge adjacent to a vertex of valence one, other edges of $T$ are interior. We in fact discard vertices of valence one at the endpoints of the legs, therefore the legs are "half-edges" having only one vertex. This can be formalized by introducing (generalized) graphs as certain sets with involutions, see [8, Definition II.5.37].

By a rooted or directed tree we mean a tree with a distinguished output leg called the root. The remaining legs are called the input legs of the tree. A tree with $n$ input legs is called an $n$-tree. A rooted tree is automatically oriented, each edge pointing towards the root. The edges pointing towards a given vertex $v$ are called the input edges of $v$, the number of these input edges is then the arity of $v$. Vertices of arity one are called unary, vertices of arity two binary, vertices of arity three ternary, etc. A tree is reduced if it has no unary vertices. A vertex $v \in \operatorname{Vert}(T)$ is an input vertex of $T$ if all its input edges are input legs of $T$. With the above conventions accepted, we must admit also the unique reduced 1-tree which consist of one edge which is both its root and the input leg, and no vertices (soul without body).

Each tree can be imbedded into the plane. By a planar tree we mean a tree with a specified (isotopy class of) imbedding. Reading off counterclockwise the input legs of a planar tree, starting from the leftmost one, gives a linear order of input legs. In the same manner, input edges of each vertex of a planar tree are linearly oriented. Vice versa, specifying linear orders of input edges of each vertex of a rooted tree $T$ uniquely determines an isotopy class of imbedding of $T$ into the plane.

Example 4.10. In Figure 3, $f$ is the root, $a$ the root vertex, $l, m, n, o, p, q, r, s, t, u$ the input legs and $g, j, k, i$ the interior edges of $T$. Vertices $b, c, d, v$ are the input vertices of $T$. The vertex $d$ is unary, $v$ binary and the remaining vertices are ternary. The tree in Figure 3 is not reduced because it contains an unary vertex. 


\section{References}

[1] V. Ginzburg and M.M. Kapranov. Koszul duality for operads. Duke Math. Journal, 76(1):203-272, 1994.

[2] V.A. Hinich and V.V. Schechtman. Homotopy Lie algebras. Advances in Soviet Mathem., 16(2):1-28, 1993.

[3] T. Lada and M. Markl. Strongly homotopy Lie algebras. Communications in Algebra, 23(6):2147-2161, 1995.

[4] T. Lada and J.D. Stasheff. Introduction to sh Lie algebras for physicists. International Journal of Theoretical Physics, 32(7):1087-1103, 1993.

[5] M. Markl. A cohomology theory for $A(m)$-algebras and applications. Journ. Pure Appl. Algebra, 83:141-175, 1992.

[6] M. Markl. Distributive laws and Koszulness. Annales de l'Institut Fourier, 46(4):307-323, 1996.

[7] M. Markl. Models for operads. Communications in Algebra, 24(4):1471-1500, 1996.

[8] M. Markl, S. Shnider, and J. D. Stasheff. Operads in Algebra, Topology and Physics, volume 96 of Mathematical Surveys and Monographs. American Mathematical Society, Providence, Rhode Island, 2002.

[9] J.-P. Serre. Lie Algebras and Lie Groups. Benjamin, 1965.

[10] J.D. Stasheff. Homotopy associativity of H-spaces I,II. Trans. Amer. Math. Soc., 108:275-312, 1963.

This article may be accessed via WWW at http://www.rmi.acnet.ge/hha/ or by anonymous ftp at

ftp://ftp.rmi.acnet.ge/pub/hha/volumes/2005/n2a7/v7n2a7.(dvi,ps,pdf)

Martin Markl markl@math.cas.cz

Mathematical Institute of the Academy

Žitná 25

11567 Praha 1

The Czech Republic 